\title{
How to select a good candidate for left atrial appendage occlusion device: case report
}

\author{
Božo Vujeva ${ }^{1 *}$, Irzal Hadžlbegović1, Boris Starčević ${ }^{2}$ \\ 'General Hospital "Dr. Josip Benčević", Slavonski Brod, Croatia \\ ${ }^{2}$ Clinical Hospital Dubrava, Zagreb, Croatia
}

\begin{abstract}
Atrial fibrillation is one of the most common arrhythmias and carries a great risk for cardioembolisation, with stroke and acute limb or mesenteric ischemia as one of the most severe consequences. Anticoagulation therapy is a gold standard therapy for embolic event protection, with new oral anticoagulants as a more attractive option for both patients and physicians. However, there are data that support the introduction of left atrial appendix occluders, like Watchman device, as a good therapeutic option for selected population of patients.
\end{abstract}

We present a case of a 73-year-old women, who was first admitted in the coronary care unit in General Hospital Slavonski Brod because of non-ST segment elevation myocardial infarction (NSTEMI). She was in sinus rhythm. She was treated with acetylsalicylic acid (ASA), clopidogrel and lowmolecular-weight heparin (LMWH), and was scheduled for a coronary angiogram during the same hospital stay. On the third day of hospitalization, before planned coronarography, she developed a large retroperitoneal hematoma with no computed tomography signs of active bleeding from any large vessel. She was treated conservatively with good recovery. During the treatment, several episodes of atrial fi-

\section{Received: $17^{\text {th }}$ Apr 2014}

"Address for correspondence: Opća bolnica "Dr. Josip Benčević", Andrije Štampara 42, HR-35000 Slavonski Brod, Croatia.

Phone: +385-35-201700

E-mail: vujevab@gmail.com brillation were documented. After introduction of amiodarone, she was in stable sinus rhythm. After partial resolution of retroperitoneal heamatoma, coronary angiography was performed showing normal coronary arteries. She was discharged home in sinus rhythm, with ASA, angiotensin-converting-enzyme inhibitor, beta-blocker, and a statin. After 2 months she presented to emergency room with clinical signs of acute left arm ischemia. Color Doppler investigation showed signs of acute closure of left axillar artery. Basal ECG showed sinus rhythm. Urgent thromboendarterectomy with a Foley catheter was performed with prompt restoration of the circulation. Early transesophageal echocardiography was performed showing a large thrombus in the left atrial appendage. Continuous ECG monitoring showed short episodes of atrial fibrillation and undulation. Along with LMWH, anticoagulation therapy with warfarin was introduced. Previous NSTEMI with normal coronary angiography and acute left arm ischemia were attributed to thromboembolisation from the left atrial appendage. Because of her history of spontaneous retroperitoneal bleeding on antiaggregation and anticoagulation therapy, she was referred to Clinical Hospital Dubrava for left atrial appendage occlusion with Watchman device.

We have discussed the appropriateness of criteria for lifetime anticoagulation therapy in atrial fibrillation and selection criteria for occlusion device implantation.

KEYWORDS: atrial fibrillation, cardioembolisation, embolic protection device.

CITATION: Cardiol Croat. 2014;9(5-6):182.

\section{Literature}

1. Reddy VY, Möbius-Winkler S, Miller MA, et al. Left atrial appendage closure with the Watchman device in patients with a contraindication for oral anticoagulation. J Am Coll Cardiol. 2013;61:2551-6. 